\title{
The Efficacy of Treatment Options to Improve Acne Vulgaris Scarring
}

\section{Noura Elgharably ${ }^{1}$ and Mohammed Al Abadie ${ }^{2}$}

${ }^{1}$ Medical School, Birmingham University, UK

${ }^{2}$ Royal Wolverhampton NHS Trust, Wolverhampton, UK

*Corresponding Author: Mohammed Al Abadie, Royal Wolverhampton NHS Trust, Wolverhampton, UK.
Received: October 08, 2021

Published: November 18, 2021

(C) All rights are reserved by Noura

Elgharably and Mohammed Al Abadie.

\section{Abstract}

A common complication of acne is scarring. This can cause severe emotional concern for patients affecting their self-image and can result in the development of insecurities. We are fortunate to have a plethora of treatment options which provide cosmetic and clinical benefit for this. We aim to review the current literature on these treatment options, with particular focus on atrophic scarring. The literature highlights the benefit and drawbacks of treatment options including lasers, radiofrequency, chemical peels, dermabrasion, subcision and fillers.

Keywords: Acne Vulgaris; Scarring; Atrophic; Laser; Dermabrasion

\section{Introduction}

Acne vulgaris is a prevalent inflammatory skin condition that affects more than $9 \%$ of the global population [1]. The pathogenesis of acne is complex and multifactorial. It includes increased sebum production, genetic propensity, androgenic stimulation, as well as bacterial flora overgrowth [2]. Adolescents are more likely to be affected $[3,4]$. Acne lesions can leave permanent scarring depending on both the severity of the condition and how long it takes to treat it. Three types of scars can present during wound healing [5]. Most commonly, there can be a loss of collagen, and this will result in the formation of pitted atrophic scarring.

Alternatively, in the case of an overproduction of collagen, elevated hypertrophic scarring can occur. There can also be overgrowth of scar tissue leading to keloidal scars.

Scarring can negatively affect patient's quality of life by causing insecurities which can have a severe emotional impact [5]. Various treatment options exist for such scars, including laser treatment, radiofrequency, micro needling, dermabrasion, chemical peels, subcision, punch methods, dermal filler, and stem cell therapy. This review looks at the application of these treatments and their effectiveness in recent literature. There are many studies in the general population however, acne is most prevalent in adolescents so this group will be the focus of the review and other groups will be alluded to as appropriate. The review also aimed to focus solely on atrophic scarring as this is the most common type.

\section{Types of scars}

Atrophic

The most prevalent type of acne scar is atrophic scarring [6]. They are hypothesised to be caused by inflammatory mediators and enzymatic degradation of collagen fibres and subcutaneous fat [6]. They clinically present as depressions in the skin because of subsequent inflammatory fibrous contractions and a regional loss of collagen. Ice pick, rolling and boxcar scars are the three forms of atrophic scars [7]. Each patient's treatment is tailored to his or her specific subtype. 
Ice pick scars (Figure 1) are deep (extending to the dermis) and narrow (less than $2 \mathrm{~mm}$ ) [9]. They account for $60-70 \%$ of atrophic scars and are often more difficult to treat due to the depth of the scar, which makes treatment methods for rolling and boxcar scars ineffective [10]. Punch excision is a common treatment that is used that creates a smaller scar which heals quickly [7,8]. The CROSS procedure (chemical reconstruction of skin scars) has also been a recent option for ice pick scars [11]. This entails dipping a syringe needle or wooden applier into concentrated trichloroacetic acid (TCA) solution and applying it locally onto the scar.

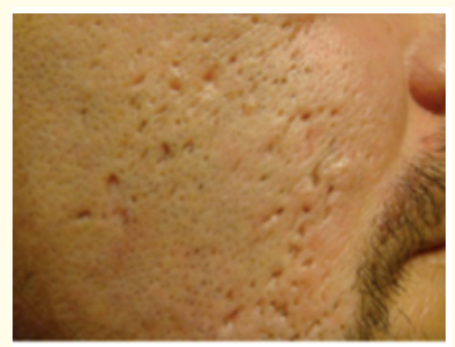

Figure 1: Icepick scar [9].

The application of TCA on the scar results in a white overlay and the induction of epidermal coagulative necrosis. This increases collagen synthesis and improves the scar [8].

Boxcar scars (Figure 2) account for 20-30\% of atrophic scars. They are wider than ice pick scars ( $1.4-4.0 \mathrm{~mm}$ ) and have defined, vertical edges [10]. Treatment is often directed with resurfacing the skin, but due to their depth they may be resistant to traditional techniques, so often this is done via chemical peels or using micro-needle [8]. The intensity of the treatment is determined by the depth as well as diameter of these scars, and risk-benefit ratio. Micro-needling uses a derma-roller or micro-needling pen, and additional platelet rich plasma (PRP) can be given topically during the procedure [12].

Rolling scars (Figure 3) are the broadest of the three subtypes (up to $5 \mathrm{~mm}$ diameter) and make up 15 - 25\% of atrophic scars [10]. They have a darkened appearance with an uneven circumference. They are triggered by fibrous adhesions which bind the dermis to the subcutis hence treatment focuses on correcting the subdermal aspect to improve the skin $[7,10]$. Subcision is beneficial for the treatment of these superficial scars [13]. It entails inserting a tiny needle into the scar on the periphery and pushing it back and forth to release the fibrous bands. The wound healing process is aided by the loosening of adhesions, which allows for bleeding and the formation of new collagen [14].

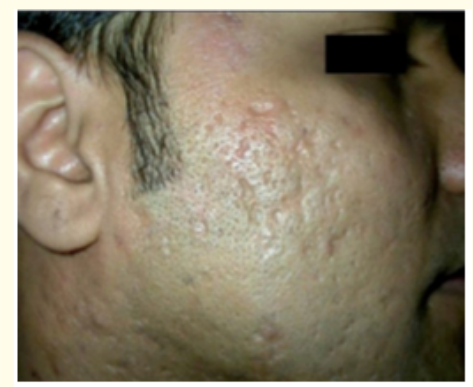

Figure 2: Boxcar scar [9].

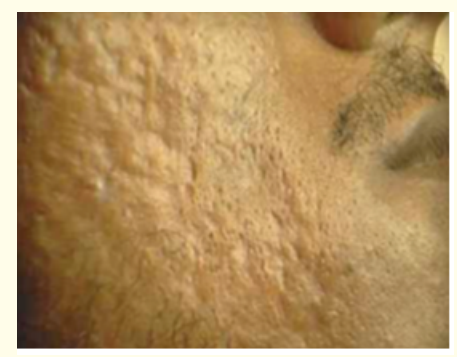

Figure 3: Rolling scar [9].

\section{Hypertrophic}

Clinically, hypertrophic scars present as hard, pink, raised lesions which do not spread into the surrounding tissue [15]. This is caused by continuous inflammation causing growth of the dermal tissue and excess fibroblastic extracellular matrix and collagen [16]. They are mostly associated with burns but may also arise after piercings, wounds, and acne [17]. Treatment options include silicone gel, 5 fluorouracil, intralesional injection of steroids, cryotherapy, the use of pulsed dye ablative and non-ablative laser and surgery [15].

\section{Keloidal}

These scars are smooth, firm, and benign growths and can also occur due to repetitive scar tissue growth. 
They generally are erythematous and outgrow the margins of the original wound overtime [17]. They present more frequently in darker skin types [16].

\section{Method}

To establish the efficacy and side effects of treatments used for acne scarring, we conducted a thorough literature search using PubMed, MEDLINE and the Cochrane Database of Systematic Reviews. We followed the PICO format with population as adolescents, the intervention was acne scarring treatment; the comparator was pulsed dye laser, radiofrequency, dermabrasion, microneedling, dermal filler, chemical peels, subcision; the outcome was patient satisfaction and grading of scar.

\section{Effectiveness of treatments}

In the clinical setting, treatment often begins with a physical examination of the patient with attention to any erythema, the type, depth and location of scarring and the patients skin type [18].

Initially clinicians begin by targeting erythema and once scarassociated erythema is tackled treatment is focused on commonly atrophic scarring or the other types of scars that are present. The gold standard for scar associated erythema is pulse dye laser (PDL) which uses thermolysis to slowly heat the oxyhaemoglobin in the microvascular parts of the dermis, destroying it to resolves erythema [19]. Two types of lasers are also used for atrophic scarring: ablative and non-ablative [20].

Nonablative laser stimulates new collagen growth and is nonwounding. Intense pulse light (IPL) is an example of this. IPL allows you to change the settings for pulse duration, fluence and filters which can be used to treat hyperpigmentation and erythema; however, it has low specificity so maximal reduction in erythema is difficult [21]. It has been compared to PDL in a randomised split face trial where both treatments provided clinical benefit however PDL had a longer lasting impact. [21,22]. In selective non-ablative treatment of acne scarring with $585 \mathrm{~m}$ pulsed dye laser, there was clinical improvement due to stimulation of collagen synthesis. Patients with deep facial acne scars received single laser treatment then later were assessed for clinical benefit. The most significant benefit was in reduction of depth of acne scars by $47.8 \%$ on average. There were no adverse effects documented in the research [22]. One separate study reported a transient side effect of erythema and purpura [21]. However, PDL has also shown efficacy to treat acne in several other studies [23-25] and has been recommended as second line therapy for linear hypertrophic scars [23]. One study commented on the combination of PDL and ablative fractional $\mathrm{CO}^{2}$ laser for immature erythematous hypertrophic scars [24].

Ablative laser is a strong laser which removes the epidermis and heats the dermis, promoting collagen fibre formation [20]. This includes the carbon dioxide laser, the erbium laser, and combinations of laser [20]. In patients treated with ablative fractional photo-thermolysis surgery there was a clinical improvement in scarring and histological evidence showed collagen and elastin synthesis in the upper and mid dermis [25].

This study highlighted a benefit of 26 - $83 \%$ in those with ablative laser in the initial month of treatment but no additional improvement beyond 3 - 6 months. The results were also quicker with fewer treatments in ablative PDL. Erythema was apparent for 3 14 days and laser resolved by 12 weeks. Patients improved by 26 - 50\% as compared to non-ablative laser, and erythema appeared for 1 - 3 days post treatment.

Despite fewer sessions for efficacy, the main concern with ablative laser treatment was patient discomfort with patients scoring 5.9 - 8.1 on the pain score, and a greater risk of post-inflammatory hyperpigmentation. It is therefore more successful than non-ablative lasers, but also more invasive and requires longer recovery time [25].

Microneedling fractional radiofrequency (MFR) has also been explored in recent literature to treat moderate to severe acne scars [26]. A retrospective study showed an $85.71 \%$ two grade improvement in the grading of the acne scarring. Patients underwent four MFR sessions with 6-week intervals between each session (Figure 4).

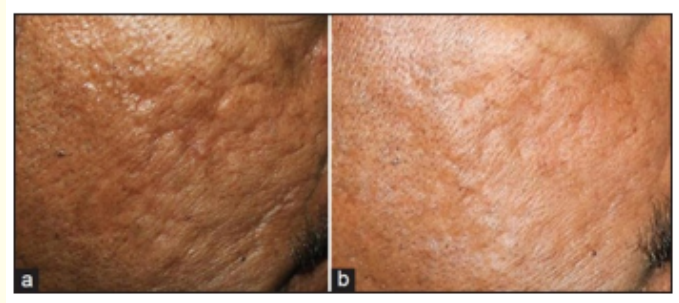

Figure 4: Improvement in acne scars after treatment with MFR [26]. 
Results were particularly effective in rolling and boxcar scars compared to ice pick scars. Mild erythema, post inflammatory hyperpigmentation, and track marks from the device were the noted side effects (Figure 5).

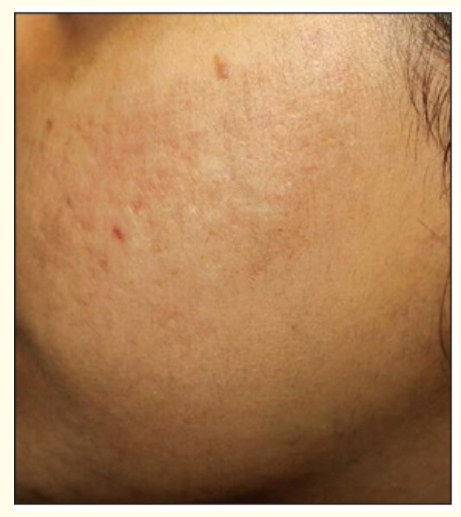

Figure 5: Track marks from the device probe after treatment [26].

The major benefit with microneedling fractional radiofrequency is the technology's ability to adjust the level of tissue disruption via varying the depth of the penetrating needle [26]. This allows different scar types to be targeted via altering needle depth and thus adds to clinical applicability.

Fractional bipolar radiofrequency can also be used to treat acne scarring. This combines radiofrequency and non-ablative laser treatment. Radiofrequency devices can be monopolar, bipolar, or fractional. A systematic review of these modalities found that microneedle bipolar radiofrequency and fractional bipolar radiofrequency devices had the best outcomes, with an expected clinical benefit of $25 \%$ to $75 \%$ after 3 to 4 sessions [27]. Although there are few side effects, the procedure itself can be associated with a temporary amount of pain and some erythema [27].

Dermabrasion is a method which removes the superficial layer of the skin via friction to reach the level of the reticular dermis [28]. This modifies the structure of the skin by activating structural proteins and which smoothen the skin's surface. It was the first important discovery in the treatment of acne scars, and can be done with sandpaper manually, or mechanically with a revolving brush or diamond fraise [28]. It is described as a safe and well tolerated procedure that produces clinical improvement in superficial scars
[29-31]. However, it is less effective in severe or deeper scars, such as ice pick and deep boxcar scars, and there is a greater risk of complications which can occur posttherapy such as discolouration and further scarring. In a recent randomised controlled trial dermabrasion was compared to fractional ablative resurfacing and despite both being effective, laser was considered safer with less downtime in recovery $[32,33]$. Dermabrasion is also operator dependent so reliant on the quality and experience of the operator otherwise there is a risk of worsening the scar [34]. The popularity of dermabrasion has also reduced due to the adverse effects related to aerosolization of blood [31].

Chemical peeling agents including salicylic acid, glycolic acid, trichloroacetic acid (TCA), phenol peel and Jessner's solution have all shown effectiveness as adjuvant treatment for acne scarring [32]. The addition of chemical peels was explored in an evidencebased review by Handog., et al. which resulted in quicker clinical improvement and as a result, higher patient satisfaction [33]. Ice pick scars were targeted with TA in two open label studies using the chemical reconstruction of skin scars (CROSS) technique. TCA was administered using a pointed applicator into the scar base in high concentrations of $65 \%$ and $100 \%$ [32]. 100\% concentration proved to be more effective, and all patients had successful results. There was one case where the effectiveness began to wane after three months, and one patient of temporary dyspigmentation [32]. In a larger study, it was also concluded that $100 \%$ concentration of TCA was deemed effective with $73 \%$ of patients achieving excellent clinical benefit in ice pick scars, and $20 \%$ receiving good improvement. There was mention of little adverse effects which included temporary hypopigmentation, erythema, and oedema during treatment [34]. TCA was also mixed with Jessner's solution in a separate study which found clinical improvement in all cases bar one who had deeper ice pick scars [34]. Chen., et al. conducted a systematic review of 12 randomised controlled trials which looked at the effectiveness of chemical peeling agents for acne vulgaris. They found that there was no significant difference between the different chemical peels used and that they were all comparable in effectiveness, but one agent was not deemed superior [35].

Dermal fillers have also been used to treat isolated atrophic rolling scars and are injected to cause a local volume increase [36]. 5 studies were explored in a systematic review by Kravvas and AlNiaimi which look at the use of poly-L-lactic acid (PLLA), collagen, polymethyl methacrylate (PMMA) and hyaluronic acid for acne 
scars [36]. In those treated with PLLA, a semi-permanent filler, there was scar reduction of 46.45 by the seventh treatment. PMMA, a permeant filler, was compared to a control saline and there was $84 \%$ patient satisfaction following therapy compared to $52 \%$. PLLA was also used for facial rolling scars in another study where $54.5 \%$ of patients reported good or excellent satisfaction. Hyaluronic acid, a temporary filler, was used in 5 patients in a separate study where less than $30 \%$ of scars were seen after 2 injection sections and monthly follow up. A more recent article by Pol., et al. Comments on a cost-effective autologous PRP intradermal filler which is beneficial for both rolling and boxcar atrophic scars [37]. The volume area and contouring of the scar was improved in a single patient case study so there is potential area for development of this agent for use (Figure 6) [37].

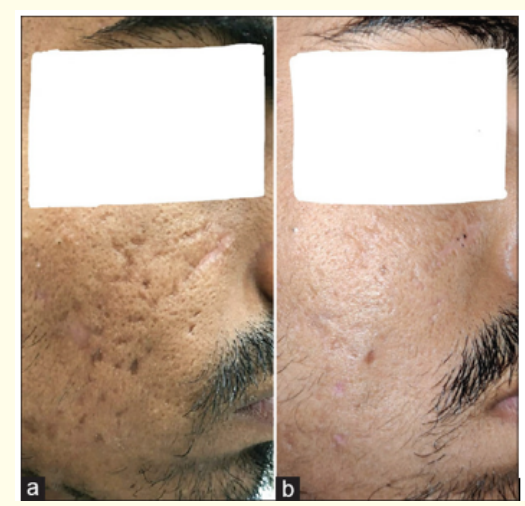

Figure 6: Improvement in rolling and box scars 5 weeks post PRP dermal filler treatment [37].

However, an issue with temporary filler is that it is not permanent, and with semi-permanent results, infection, pain, and erythema can also occur [38].

Punch excision is a common technique used for individual atrophic ice pick scars $[6,7]$. This involves creating a new scar by the punch excision which heals much quicker than a normal scar [6]. Scars should be $4-5 \mathrm{~mm}$ apart when being treated at the same time, according to Jacob., et al. or the risk of high tension will result in poor healing [38]. Treatments every four weeks were also recommended, which resulted in the best clinical outcome [39].

\begin{tabular}{|c|c|c|}
\hline Technique & Scar subtype & Side effects of technique \\
\hline Pulse dye laser & $\begin{array}{l}\text { Scar associated } \\
\text { erythema }\end{array}$ & $\begin{array}{l}\text { Rarely reported erythema } \\
1-3 \text { days post treatment }\end{array}$ \\
\hline $\begin{array}{l}\text { Non-ablative } \\
\text { laser }\end{array}$ & $\begin{array}{l}\text { All subtypes, } \\
\text { hypertrophic } \\
\text { scars }\end{array}$ & $\begin{array}{c}\text { Hyperpigmentation, ery- } \\
\text { thema }\end{array}$ \\
\hline Ablative laser & $\begin{array}{l}\text { All subtypes, } \\
\text { hypertrophic } \\
\text { scars }\end{array}$ & $\begin{array}{l}\text { Higher risk of pain, erythe- } \\
\text { ma 3-14 days post treat- } \\
\text { ment, post- inflammatory } \\
\text { hyperpigmentation. }\end{array}$ \\
\hline $\begin{array}{l}\text { Microneedle } \\
\text { fractional ra- } \\
\text { diofrequency }\end{array}$ & $\begin{array}{l}\text { Rolling, boxcar } \\
\text { scars }\end{array}$ & $\begin{array}{l}\text { Mild erythema and post } \\
\text { inflammatory hyperpigmen- } \\
\text { tation, track marks }\end{array}$ \\
\hline $\begin{array}{l}\text { Fractional bi- } \\
\text { polar radiofre- } \\
\text { quency }\end{array}$ & All subtypes & $\begin{array}{c}\text { Temporary pain and ery- } \\
\text { thema }\end{array}$ \\
\hline Dermabrasion & Rolling & $\begin{array}{c}\text { Further scarring, hyper- } \\
\text { pigmentation, operator } \\
\text { dependent }\end{array}$ \\
\hline Chemical peel & Ice pick & $\begin{array}{l}\text { temporary hypopigmenta- } \\
\text { tion, erythema, oedema }\end{array}$ \\
\hline Dermal Filler & Rolling, boxcar & $\begin{array}{l}\text { Semi-permanent, infection, } \\
\text { pain, erythema }\end{array}$ \\
\hline Punch Excision & Ice pick & Risk of poor heeling \\
\hline
\end{tabular}

Table 1

Summary of techniques

Time to treatment

Early detection and treatment of acne lesions is critical in minimising scarring $[6,18]$. According to a study of acne patients' beliefs and perceptions, $16 \%$ of acne patients seek treatment, and $74 \%$ do not seek treatment for more than 12 months, $12 \%$ do not for up to 6 - 12 months, $6 \%$ for up to 6 months, and shockingly, 7 percent wait less than 3 months before seeking professional help [40]. Thus, it is crucial to think about the relationship between severity and length of inflammation in scar creation and question why patients chose not to seek treatment for acne scars sooner.

\section{Discussion}

Acne vulgaris can result in many different types of scar formation. Despite its widespread occurrence, there is not a superior treatment option for tackling all these scar types, and often multiple methods are encouraged depending on the type of scar, side effects and medical professional. The variety of treatments avail- 
able each have their own set of benefits and drawbacks with many causing transient erythema, pain or dyspigmentation.

For example, ablative PDL are particularly useful for atrophic scarring but can cause pain and erythema. Non ablative PDL is mostly beneficial on boxcar scars but has lower efficacy compared to ablative. Microneedling is a minimally invasive technique and particularly effective when used as a dual therapy with PRP. However, the future of this technique is unclear as further studies must be conducted to provided clear significance and benefit of use.

Ice pick scars are often targeted using chemical peels however these are accompanied with a considerable downtime and not always effective so may also be targeted with PDL. Rolling scars are quite difficult to treat with subcision and filler regarded as common options for this scar subtype.

The studies discussed vary with sample size, format, follow up time and demographics. Different outcome measures were used with regards to patient satisfaction or grading scar types, thus there is an element of bias, particularly subjective patient bias, so a potential area for inconsistency between the data. This makes it difficult to draw thorough conclusions from the report.

\section{Conclusion}

To summarise, scarring after acne is very prevalent and treating it remains a challenge with no single conclusive treatment. PDL, microneedling, dermabrasion, chemical peels and fillers have been employed with varied success, but all prove to have some clinical benefit. Some techniques are more appropriate for certain scar subtypes, and all have the potential for negative side effects. Likewise, to many aspects of medicine, treatment must be tailored specifically for each patient combining all relevant factors.

\section{Bibliography}

1. Tan JK and Bhate K. "A global perspective on the epidemiology of acne". British Journal of Dermatology 172.1 (2015): 3-12.

2. Toyoda M and Morohashi M. "Pathogenesis of acne". Medical Electron Microscopy 34.1 (2001): 29-40.

3. Whiting DA Acne. "Guidelines of care for acne vulgaris management”. The Western Journal of Medicine131.6 (1979): 551557.
4. "Acne Vulgaris: management". NICE guidelines [NG198] (2021).

5. Magin P., et al. "Psychological sequelae of acne vulgaris: results of a qualitative study". Canadian Family Physician 52 (2006): 978-979.

6. Goodman GJ. "Post-acne scarring: a review of its pathophysiology and treatment”. Dermatological Surgery 26.9 (2000): 857871.

7. NHS. Acne: Complications (2021).

8. Levy LL and Zeichner JA. "Management of acne scarring, part II: a comparative review of non-laser-based, minimally invasive approaches". American Journal of Clinical Dermatology 13 (2012): 331-40.

9. Kannangara AP. "Acne scar management". Global Dermatology (2015): 2.

10. Connolly D., et al. "Acne Scarring-Pathogenesis, Evaluation, and Treatment Options". The Journal of Clinical and Aesthetic Dermatology 10.9 (2017): 12-23.

11. Fabbrocini G., et al. "CROSS technique: chemical reconstruction of skin scar method". Dermatology Therapy 21 (2008): S29-S32.

12. Callaghan DJ. "Review on the treatment of scars". Plastic And Aesthetic Research 7 (2020): 66.

13. Aalami Harandi S and Balighi K. "Subcision-suction method: a new successful combination therapy in treatment of atrophic acne scars and other depressed scars". The Journal of the European Academy of Dermatology and Venereology 25 (2011): 92-99.

14. Chandrashekar B and Nandini A. "Acne scar subcision". Journal of Cutaneous and Aesthetic Surgery 3.2 (2010): 125-126.

15. English RS and Shenefelt PD. "Keloids and hypertrophic scars". Dermatological Surgery 25.8 (1999): 631-638.

16. Gauglitz GG., et al. "Hypertrophic scarring, and keloids: pathomechanisms and current and emerging treatment strategies". Molecular Medicine 17.1-2 (2011): 113-125.

17. Wang ZC., et al. "The Roles of Inflammation in Keloid and Hypertrophic Scars". Frontiers in Immunology 11 (2020): 603187. 
18. "Acne vulgaris: evidence reviews for management of acne vulgaris-associated scarring". National Institute for Health and Care Excellence 15 (2021).

19. Alster TS and McMeekin TO. "Improvement of facial acne scars by the $585 \mathrm{~nm}$ flashlamp-pumped pulsed dye laser". Journal of the American Academy of Dermatology 35.1 (1996): 79-81.

20. Laser resurfacing - Mayo Clinic - Mayo Clinic Organisation (2021).

21. YS Suh HS., et al. "Intense pulsed light vs. pulsed-dye laser in the treatment of facial acne: a randomized split-face trial". The Journal of the European Academy of Dermatology and Venereology 24.7 (2010): 773-780.

22. Patel Nita and Clement. "Selective Nonablative Treatment of Acne Scarring With 585 nm Flashlamp Pulsed Dye Laser". Dermatologic Surgery: Official Publication for American Society for Dermatologic Surgery 28 (2002): 942-945.

23. Marini L. "Laser- and RF-based treatments for acne vulgaris: newinteresting options a commentary". The Journal of the European Academy of Dermatology and Venereology 32 (2018): 514-514.

24. Orringer JS., et al. "A randomized, controlled, split-face clinical trial of 1320-nm Nd:YAG laser therapy in the treatment of acne vulgaris". Journal of the American Academy of Dermatology 56.3 (2007): 432-438.

25. Ong Michal and Bashir Saqib. "A Systematic Review of Laser Resurfacing for Acne Scars". Journal of the American Academy of Dermatology 66 (2012): 10.

26. Chandrashekar., et al. "Evaluation of microneedling fractional radiofrequency device for treatment of acne scars". Journal of Cutaneous and Aesthetic Surgery 7.2 (2014): 93-97.

27. Simmons BJ., et al. "Use of radiofrequency in cosmetic dermatology: focus on nonablative treatment of acne scars". Clinical Cosmetic and Investigational Dermatology 7 (2014): 335-339.

28. Hession MT and Graber EM. "Atrophic acne scarring: a review of treatment options". The Journal of Clinical and Aesthetic Dermatology 8.1 (2015): 50-58.

29. Christophel JJ., et al. "A randomized controlled trial of fractional laser therapy and dermabrasion for scar resurfacing". Dermatological Surgery 38 (2012): 595-602.
30. Aronsson A., et al. "Effects of dermabrasion on acne scarring. A review and a study of 25 cases". Acta Dermatology -Venereologica 77.1 (1997): 39-42.

31. Minkis A. "Microdermabrasion: Background, History of the Procedure, Problem". Medline (2021).

32. Lee JB., et al. "Focal treatment of acne scars with trichloroacetic acid: chemical reconstruction of skin scars method". Dermatological Surgery 28.11 (2002): 1017-1021.

33. Handog EB., et al. "Chemical peels for acne and acne scars in asians: evidencebased review". Journal of Cutaneous and Aesthetic Surgery 5.4 (2012): 239-246.

34. Al-Waiz MM and Al-Sharqi AI. "Medium-depth chemical peels in the treatment of acne scars in dark-skinned individuals". Dermatological Surgery 28 (2002): 383-387.

35. Chen X., et al. "Chemical peels for acne vulgaris: a systematic review of randomised controlled trials". BMJ Open 8 (2018): e019607.

36. Narins RS and Bowman PH. "Injectable skin fillers". Clinics in Plastic Surgery 32 (2005): 151-162.

37. Kravva G and Al-Niaimi F. "A systematic review of treatments for acne scarring. Part 1: Non-energy-based techniques". Scars Burns and Healing 3 (2017).

38. Pol D., et al. "A novel cost-effective autologous dermal filler for atrophic acne scar". Indian Dermatol Online Journal 12 (2021): 361-362.

39. Jacob CI., et al. "Acne scarring: a classification system and review of treatment options". Journal of the American Academy of Dermatology 45 (2001): 109-117.

40. Tan JK., et al. "Beliefs and perceptions of patients with acne". Journal of the American Academy of Dermatology 44.3 (2001): 439-445.

\section{Volume 3 Issue 12 December 2021 CAll rights are reserved by Noura Elgharably and Mohammed Al Abadie.}

